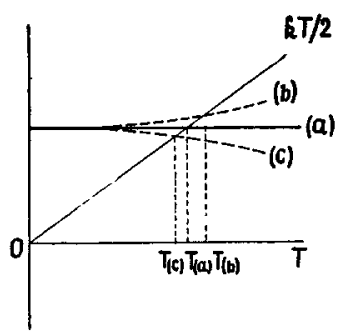

Fig. 1. Influence of excess volume on an U.C.P., from relation (11). (a) $g_{e}$ in regular solutions $\left(v_{0}=0\right)$, (b) $g_{e}$ when contraction on mixing $\left(v_{e}<0\right)$. (c) $g_{e}$ when expansion on mixing $\left(v_{e}>0\right)$.

may be put approximately in the form

$$
h_{e}=K_{h} x_{A} x_{B}, \quad T s_{c}=K_{s} x_{A} x_{B},
$$

owing to relations (3) and (4). $K_{h}$ and $K_{s}$ are independent of $x_{A}$ and $x_{B}$ but depend on temperature. From (9), the excess free energy becomes

$$
g_{e}=\left(K_{h}-K_{s}\right) x_{A} x_{B}
$$

At the critical point, from relations (5) and (10), we get

$$
x_{A c}=x_{B c}=\frac{1}{2}, \quad k T_{c}=\left(K_{h}-K_{s}\right) / 2=2 g_{e}\left(T_{c}, 0.5\right) .
$$

Relation (11) is very useful for a qualitative discussion : the critical temperature, if it exists, is given by the intersection of $k T / 2$ and $g_{e}(T, 0.5)$ on a graphic.

(A) Upper critical solution phenomena occur in endothermic mixtures. There are two possibilities:

(a) There is a contraction on mixing. Then, from (3) and (4), $g_{e}$ is higher than in the strictly regular solutions theory. Consequently, according to (11), the U.C.P. will be higher than in regular solutions. See Fig. 1. Exact calculations were made by taking the complete form of $g_{e}$ in the case called "dispersion forces"2 $\left(\theta \simeq-\delta^{2} / 8\right)$. The critical temperature is

$$
k T_{c} / z\left|\epsilon_{A A}\right|=0.090 \delta^{2}\left[1+0.36 \delta^{4}(1+\delta / 2)^{-3}\right],
$$

while in strictly regular solutions it was

$$
k T_{c} / z\left|\epsilon_{A A}\right|=0.090 \delta^{2} .
$$

The critical concentration is no more equal to 0.5 but is somewhat nearer to constituent $A$. Equations (12) and (13) are plotted on Fig. 2. Similar results have been obtained from another cell model, namely the harmonic oscillator model. ${ }^{1}$

(b) There is an expansion on mixing. Then, according to (3), (4), and (11), the U.C.P. will be lower than in the regular solutions theory. See Fig. 1. Exact calculations, made in the case called "association in inert solvent," show that the $T_{c}$ is

$$
k T_{c} / z\left|\epsilon_{A A}\right|=0.359 \delta\left(1-1.8 \delta+2.8 \delta^{2}\right) \text { for } \delta \leqslant 0.3 \text {, }
$$

while for regular solutions,

$$
k T_{c} / z\left|\epsilon_{A A}\right|=0.359 \delta \text {. }
$$

These two equations are plotted on Fig. 3. The results are compared to the quasi-chemical correction. The critical concentration is somewhat nearer to constituent $B$.

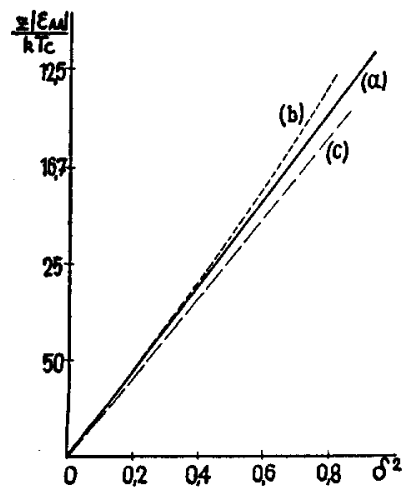

FIG. 2. Dispersion forces. Upper critical temperature in function of $\delta^{2}$. (a) Strictly regular solutions; (b) cell model, taking into account the contraction on mixing (c) quasi-chemical approximation

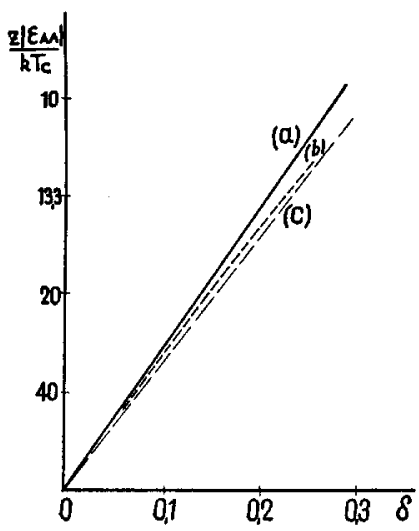

Fig. 3. Association in inert solvent. Critical temperature in function of $\delta$. (a) Strictly regular solutions, (b) cell model taking polume expansion, (c) quasi

(B) From relations (7) and (8), it follows that a L.C.P. can only occur in exothermic mixtures $\left(h_{m}<0\right)$, where $s_{e}<-s^{*}$. This last condition cannot be filled at any reasonable temperature for the liquid state, even for large values of $\delta$ and $\theta$. Such a model cannot give rise to a L.C.P., which must be related to other factors and especially to anisotropic interactions between molecules. These conclusions agree with those of Rowlinson ${ }^{5}$ and reverse those of Ono, ${ }^{6}$ who thought that a L.C.P. could occur in such simple solutions.

We are much indebted to Professor Prigogine, who suggested the present work, for constant interest and advice.

1 I. Prigogine and G. Garikian, Physica 16, 239 (1950)

2 I. Prigogine and V. Mathot, J. Chem. Phys. 20, 49 (1952).

3 I. Prigogine and R. Defay, Thermodynamique chimique (Desoer, Liége, 1950), new edition.

O. K. Rice, Chem. Rev, 44, 65 (1949)

J. S. Rowlinson, Proc. Roy. Soc. (London) A214, 192 (1952).

6 S. Ono, Mem. Faculty Eng. Kyushu Imp. Univ. 12, 201 (1950).

\title{
Interaction of Methylene Deformation Frequencies in Paraffin Crystals*
}

R. S. STEIN, Department of Chemistry, University of Massachusetts, Amherst, Massachusetts

$$
\text { AND }
$$
G. B. B. M. SutherLand, Department of Physics, University of Michigan,

(Received December 11, 1952)

$\mathrm{T}$

$\mathrm{HE}$ doublet at $14 \mu$ in the spectra of polythene and other polymethylene hydrocarbons has been discussed in several papers. ${ }^{1-3} \mathrm{We}$ should like to report the results of some investigations regarding the nature of this doublet which we have conducted and which will be reported in detail together with some more recent work in a forthcoming publication. We find that:

(1) The doublet consists of two components, one at $720 \mathrm{~cm}^{-1}(A)$ and one at $730 \mathrm{~cm}^{-1}(B)$

(2) In all samples of unoriented polythene which we have studied, component $(A)$ is more intense than $(B)$. Component $(B)$ is relatively more intense in samples having higher crystallinity.

(3) The ratio of the intensity of component $(B)$ to that of $(A)$ gradually decreases with increasing temperature in a manner similar to the decrease in crystallinity with temperature.

(4) The relative intensity of band $(B)$ decreases appreciably on stretching (decreasing approximately to half in a 400 percent elongation).

(5) Both bands absorb most strongly with radiation polarized perpendicular to the direction of stretching the sample. The dichroic ratio for band $(B)$ is much greater than that for $(A)$.

$(6)$ On cooling to liquid nitrogen temperature band $(B)$ became. appreciably sharper and shifted from 730 to $733 \mathrm{~cm}^{-1}$. Band $(A)$ was not appreciably affected.

(7) The spectra of a sample of a completely deuterated paraffin revealed that the counterpart of band $(A)$ shifted to $518 \mathrm{~cm}^{-1}$ and 
band $(B)$ to $524 \mathrm{~cm}^{-1}$, both bands decreasing in frequency in approximately the ratio $1 / \sqrt{2}$. The counterpart of the $6.8 \mu$ band in polythene is also a doublet in the deuterated paraffin with two components at 1086 and $1093 \mathrm{~cm}^{-1}$. This observation led to a reexamination of the $6 \mu$ band in polythene, which revealed that this is a doublet consisting of two components at about 1464 and 1473 $\mathrm{cm}^{-1}$. The higher frequency component disappears on heating. This doublet changes with crystallinity, stretching polarization and temperature in a manner analogous to that of the $6 \mu$ doublet.

(8) An examination of a series of pure crystalline $n$-paraffins ranging from $\mathrm{C}_{18} \mathrm{H}_{38}$ to $\mathrm{C}_{54} \mathrm{H}_{110}$ revealed that the $14 \mu$ doublet is present in their spectra, with both components of equal intensity. On heating, component $(B)$ disappeared sharply at a temperature corresponding to the transition temperature for these hydrocarbons identical with the temperature found by $\mathbf{x}$-ray and specific heat methods.

All of these observations are consistent with the explanation that the spectra of polythene consists of a super-position of spectra of the crystalline and amorphous portions. The crystallites are oriented to a higher degree on stretching. The doublets occur only in the crystallite spectra in which case both components are of equal intensity in the unstrained crystallite. It can be shown ${ }^{4}$ that splittings of this type may arise from coupling between normal vibrations of the $\mathrm{CH}_{2}$ groups in adjacent hydrocarbon chains in the crystal lattice of a normal paraffin. Component $(B)$ results from deformations in phase with those of nearest neighbors, while component $(A)$ results from out-of-phase deformations. The magnitude of the separation of the components depends on the distance of closest approach of nearest neighbor hydrogens and on the degree of van der Waals interaction.

* This work was carried out while both authors were at the Department of Colloid Science, Cambridge University, England, 1948-1949. Mr. Stein's work was supported in part by a fellowship from the National Research Council.

1 Elliot, Ambrose, and Temple, J. Chem. Phys. 16, 877 (1948)

${ }^{2} \mathrm{H}$. W. Thompson and P. Torkington, Proc. Roy. Soc. (London) 184A, 3 (1945)

King, Hainer, and McMahar, J. Appl. Phys. 20, 559 (1949).

${ }^{4}$ Stein and Montroll (to be published).

Investigation of the Heat of Vaporization of Carbon

William A. Chupka, Mallinckrodt Chemical Laboratory, Harvard University, Cambridge, Massachuseits

AND

Mark G. Inghram, University of Chicago and Argonne National Laboralory, Chicago, Illinoi

(Received December 1, 1952)

$\mathrm{T}$

$\mathrm{HE}$ heat of vaporization of carbon is in considerable controversy. Three possible spectroscopic values for the dissociation energy of $\mathrm{CO}$ coupled with well established thermodynamic data give three possible values for this quantity. The three values are $136 \mathrm{kcal} / \mathrm{mole}, 141 \mathrm{kcal} / \mathrm{mole}$, and $170 \mathrm{kcal} / \mathrm{mole}$. The controversy revolves around two questions; the accommodation coefficient for evaporation, and the identity and relative amounts of the evaporating species. An accommodation coefficient near unity, assuming the evaporation occurs largely as $C$, would favor the highest value, according to available data on the rate of evaporation from a graphite surface. Doehaerd et al. ${ }^{1}$ claim that the accommodation coefficient is actually about 0.001 . If this is true, one of the lower heats of evaporation is correct. One of the above authors, Waelbroeck, claims that the major part of the species evaporating from a surface is $\mathrm{C}_{2}$. This is in contrast with Brewers determination ${ }^{2}$ where he finds the $\mathrm{C}_{2}$ evaporation to be of the order of 0.0001 of the total evaporation. The main objection of Brewer to the experiments of Doehaerd et al. is the possibility of insufficient outgassing of their graphite sample.

One possible method of resolving these difficulties is to use a mass spectrometer to identify and measure the relative amounts of the various species evaporated from a graphite surface as a function of surface temperature. We have made preliminary measurements using this technique.

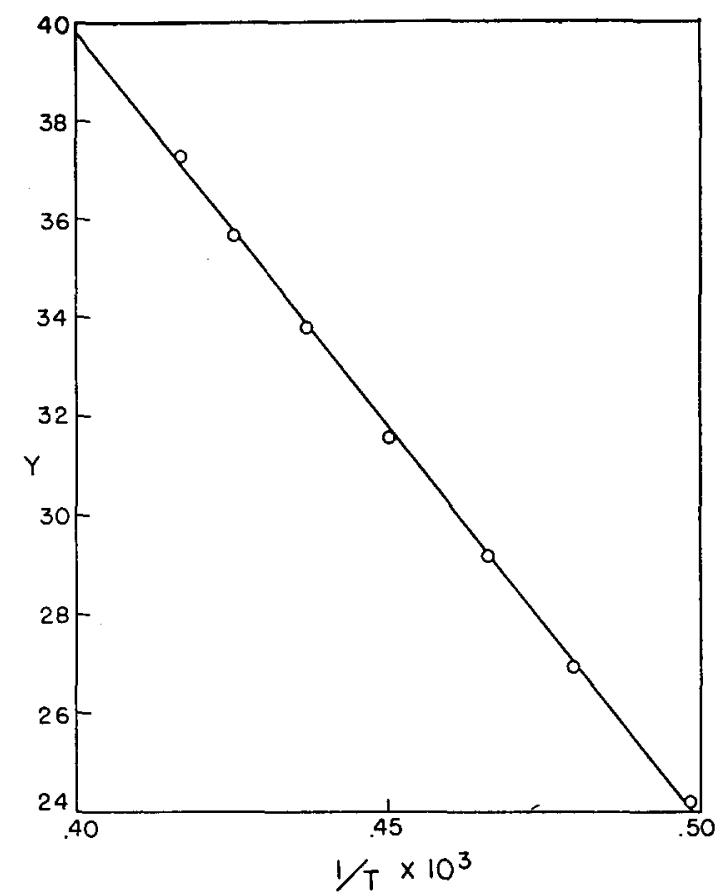

FIG. 1. Rate of evaporation of carbon atoms as a function of temperature. $Y=R \ln I\left(\mathrm{C}^{+}\right) T-2 \Delta C_{p} 1000 / T+\Delta C_{p} \ln 1000 / T$, where $I\left(\mathrm{C}^{+}\right)$is the $\mathrm{C}^{+}$ion beam intensity and $\Delta C_{p}=-3.2 \mathrm{cal} / \mathrm{g}$ atom degree. $I\left(C^{+}\right) T$ is proportional to the pressure of carbon atoms since $I\left(\mathrm{C}^{+}\right)$is proportional to the number of atoms crossing and the time spent in the ionization region. Slope of line $=-\Delta H\left(2000^{\circ} \mathrm{K}\right)$ (apparent) of the process $\mathrm{C}$ (graphite) $\rightarrow \mathrm{C}\left({ }^{3} P\right)$ (gas).

The technique used consisted of bombarding the constituents evaporated from a carbon filament with controlled energy electrons and determining the mass of the ion produced with a standard single focusing magnetic analyzer. The constituents were thus identified both by mass and by appearance potential. The $\mathrm{C}^{+}$ion appeared at an electron bombarding energy of $11.1 \pm 0.5$ volts, the $\mathrm{C}_{2}{ }^{+}$appeared at $11.5 \pm 1$ volts. The 11.1 volt appearance potential for $\mathrm{C}^{+}$ion indicates that the evaporation of $\mathrm{C}$ occurs in the ground state. This assumes that the half-life of the excited ${ }^{5} S$ state is longer than the transit time between evaporation and ionization. The time of transit is about $5 \times 10^{-6} \mathrm{sec}$ while the half-life of the ${ }^{5} S$ state is estimated to be of the order of $10^{-4}-10^{-5} \mathrm{sec}$ from Shenstone's ${ }^{3}$ line intensities.

In addition to mass resolution and appearancepotentials, the ion beams were studied by retarding potential techniques. This served to show whether the evaporating species have kinetic energy in excess of thermal energies. According to a theory of Herzberg carbon atoms may evaporate over a potential barrier which would give resulting atoms an excess kinetic energy in an amount equal to the difference between the heat of sublimation and the activation energy. Any excess kinetic energy would have to be subtracted from the apparent heat of vaporization obtained by temperature plots of evaporation from a surface in order to get the true heat of sublimation.

The variation of carbon atom evaporation with temperature is shown in Fig. 1. The slope yields an apparent $\Delta H\left(2000^{\circ} \mathrm{K}\right)$ of sublimation of $179.8 \pm 10 \mathrm{kcal} / \mathrm{mole}$ by a least squares analysis. This gives a $\Delta H\left(0^{\circ} \mathrm{K}\right)$ of $178.5 \pm 10 \mathrm{kcal} / \mathrm{mole}$. This value agrees fairly well with the high spectroscopic value and eliminates the lower values as activation energies of sublimation.

Measurements of the energy of the $\mathrm{C}^{+}$ions showed them to have thermal energies within \pm 0.3 volt. This value is incompatible with Herzberg's suggestion of a potential barrier of the order of 1.5 volts. On the other hand, the proposal of Herzberg, Herzfeld, and Teller ${ }^{5}$ is not affected by this observation so that a low accommodation coefficient is still possible. 